\title{
Transmission Electron Microscopy Study of Metallic Nanowires Fabricated from Mesoporous Silica Templates
}

\author{
W.L. Zhou ${ }_{* * * *}^{*}$, D. Wang ${ }_{* * *}^{* *}$ B.F. McCaughy ${ }^{* *}$, J.E. Hampsey ${ }^{* *}$, X. Ji ${ }^{* *}$, Y.B. Jian ${ }^{* * *}$, H. Xu ${ }^{* * *}$, C.J. \\ Brinker $^{* * * *}$, and Y. Lu
}

*Advanced Materials Research Institute, University of New Orleans, New Orleans, LA 70148

${ }^{* *}$ Dept. of Chemical Engineering, Tulane University, New Orleans, LA 70118

${ }^{* * * *}$ Dept. of Earth and Planetary Sciences, The University of New Mexico, Albuquerque, NM 87131

${ }^{* * * * *}$ Sandia National Laboratory and Dept. of Chemical and Nuclear Engineering, University of New Mexico, Albuquerque, NM 87131

Several methods such as e-beam lithography, step-edge decoration, and templated growth have been developed to fabricate metallic nanostructured materials. The hard templating approach is conceptually simple to implement, however, the use of porous alumina or polycarbonate membranes as templates usually results in polycrystalline nanowires or nanowires with large diameters (20-1000 nm), which may preclude quantum confinement effects. In this paper we present a TEM study of Pd nanowires fabricated from mesoporous silica templates.

The Pd nanowires were fabricated using an electro-deposition method [1]. Conventional transmission electron microscopy (TEM) and high resolution TEM observation were performed with a JEOL 2010 equipped with DX Prime EDS microanalysis system. Electron energy loss spectroscopy (EELS) were obtained on a JEOL 2010F FASTEM with a Gatan GIF system.

Fig. 1a shows a representative TEM image of palladium nanowires. The diameters of nanowires observed by TEM range from 4-8 $\mathrm{nm}$ with an average diameter of $7 \mathrm{~nm}$. This corresponds to the pore diameters of the silica template $(5 \mathrm{~nm}$ to $9 \mathrm{~nm})$ determined by nitrogen adsorption measurements. Fig. $1 \mathrm{~b}$ shows a single nanowire obtained by sonic dispersion. The inset shows a $<110>$ nanodiffraction pattern of the nanowire. It indicates the formation of local single crystalline nanowires with a cubic structure. Fig.1c is a HRTEM image of a single Pd nanowire. The nanowire is about $4 \mathrm{~nm}$ and the (111) lattice across the nanowires with interplanar spacing of $0.23 \mathrm{~nm}$, similar to that of bulk Pd. The nanowires formed a swirling mesostructure after the removal of silica as shown in Fig.1d. Both the diffraction and EDS analysis did not show any PdO on the surface. Fig. 2 shows the EELS spectra of a Pd nanowires, metallic Pd reference, and $\mathrm{PdO}$ reference. The $\mathrm{Pd}$ nanowire and Pd reference show similar EELS spectra. Comparing the spectrum of the $\mathrm{Pd}$ nanowires with that of $\mathrm{PdO}$, the absence of oxygen K-edge peaks in the spectrum of $\mathrm{Pd}$ nanowire indicates the absence of $\mathrm{PdO}$ on the $\mathrm{Pd}$ nanowire surface. The $\mathrm{Pd} \mathrm{M}_{4,5}$ edge peaks are labeled as A,B, and $\mathrm{C}$ in Fig. 2 The shift of these peaks to higher energy in the $\mathrm{PdO}$ spectrum is due to the oxidation of $\mathrm{Pd}$.

\section{References}

[1] D. Wang, W.L. Zhou, B.F. McCaughy, J.E. Hampsey, X.Ji, Y.B. Jiang, H.Xu, J. Tang, R.H. Schmehl, C.J. O'Connor, C.J. Brinker, and Y, Lu, Adv. Mater. 15, (2003) 130.

[2] The authors gratefully acknowledge the support of research grant of Louisiana Board of Regions Contract No. MSF/LEQSF (2001-04)-RII-03. 


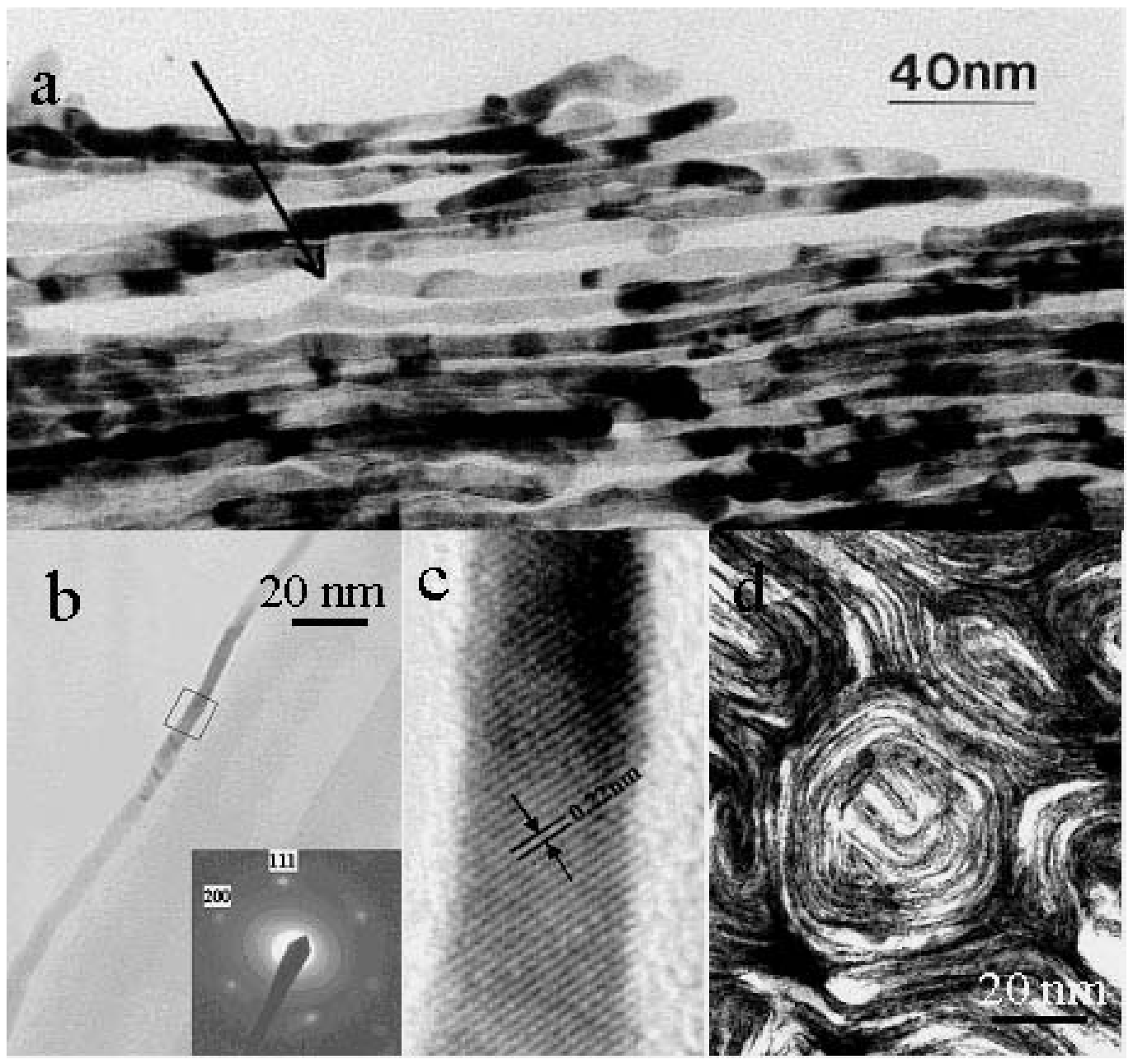

Fig.1 TEM images of Pd nanowires. a) Electrodeposition Pd nanowires. b) A single nanowire. The inset is the nanodiffraction pattern of the Pd nanowire. c) HRTEM image of Pd nanowire. d) Swirling mesostructure of Pd nanowire.

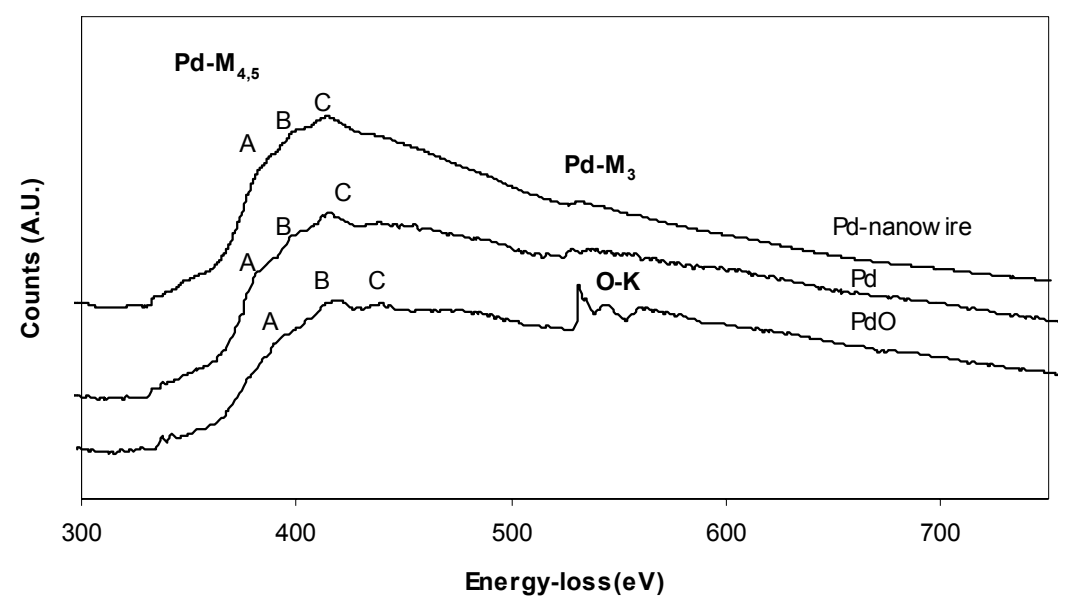

Fig.2 EELS spectra of Pd nanowire, metallic Pd and PdO. 